Published in final edited form as:

Pinchasik, B.-E.-S., Steinkühler, J., Wuytens, P. C., Skirtach, A. G., Fratzl, P., \& Möhwald, H. (2015). From Beetles in Nature to the Lab: Actuating Underwater Locomotion on Hydrophobic Surfaces. Langmuir, 31(51), 13734-13742. doi:10.1021/acs.langmuir.5b03821.

\title{
From Beetles in Nature to the Lab: Actuating Underwater Locomotion on Hydrophobic Surfaces
}

\author{
Bat-El Pinchasik, Jan Steinkühler, Pieter Wuytens, Andre G. Skirtach, Peter Fratzl and \\ Helmuth Möhwald
}

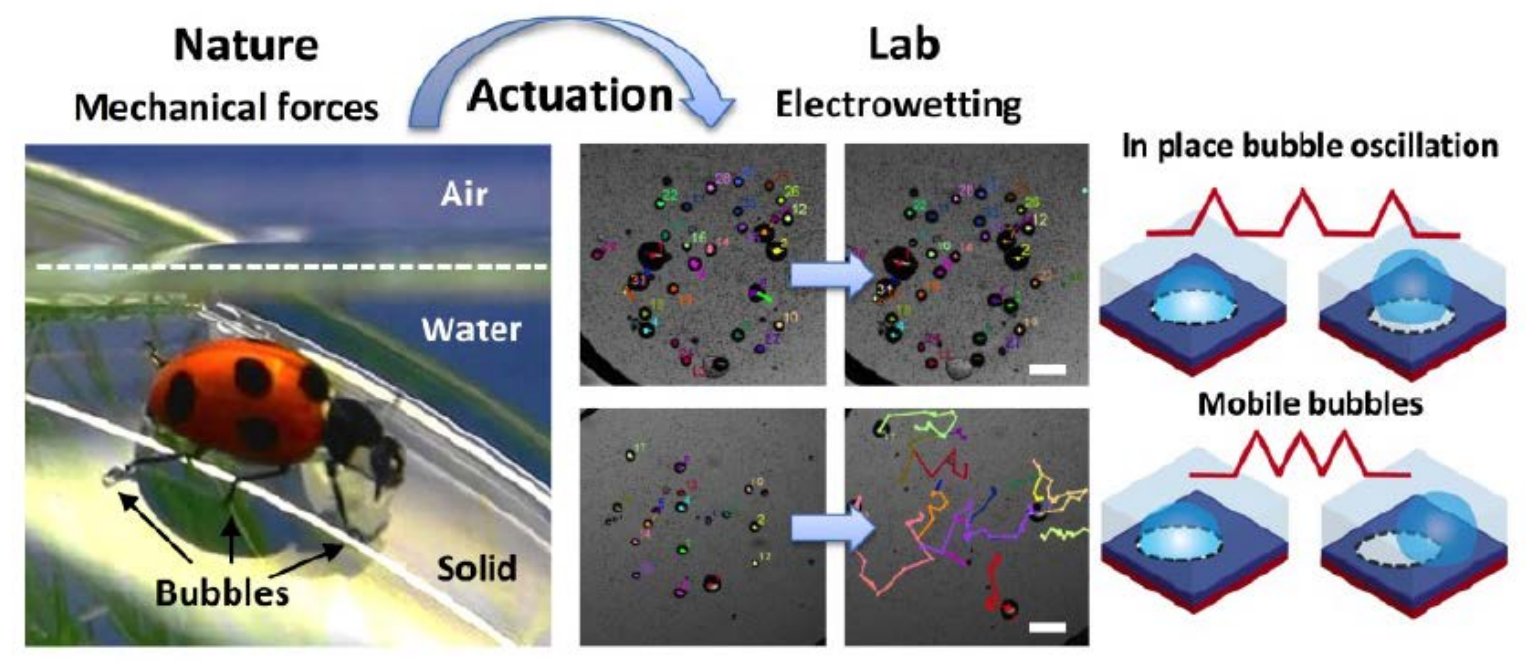

Bubbles take a random walk underwater. Two cases of bubbles' radial oscillation patterns: bubble oscillation in place (top) and bubble free motion on a hydrophobic surface (bottom). Right: optical micrographs of bubbles on a surface showing the two different behaviors along 5500 oscillation cycles. Scale bar conesponds to $1 \mathrm{~mm}$. Figure of the ladybird reproduced with permission from Natme Publishing Group. 


\section{From Beetles in Nature to the Lab: Actuating}

\section{Underwater Locomotion on Hydrophobic Surfaces}

Bat-El Pinchasik*, ${ }^{*}$ Jan Steinkühler, ${ }^{2}$ Pieter Wuytens, ${ }^{3}$ Andre G. Skirtach, ${ }^{3,4}$ Peter Fratzl ${ }^{1}$ and Helmuth Möhwald ${ }^{5}$

${ }^{1}$ Department of Biomaterials, Max-Planck Institute of Colloids and Interfaces, Golm/Potsdam, D14476 Germany

${ }^{2}$ Department of Theory and Bio-systems, Max-Planck Institute of Colloids and Interfaces, Golm/Potsdam, D14476 Germany

${ }^{3}$ Photonics Research Group, INTEC Department, Ghent University - IMEC

${ }^{4}$ Department of Molecular Biotechnology \& NB-Photonics, Ghent University, 9000 Ghent, Belgium

${ }^{5}$ Emeritus Group of Interfaces, Max-Planck Institute of Colloids and Interfaces, Golm/Potsdam, D14476 Germany

*Address correspondence to: batel.pinchasik@mpikg.mpg.de

\section{KEYWORDS}

Hydrophobic-hydrophilic, Janus nanoparticles, Microbubbles, Electrowetting, Insects, Stochastic motion 


\begin{abstract}
Controlled wetting and de-wetting of surfaces is a primary mechanism used by beetles in nature, such as the ladybird and the leaf beetle for underwater locomotion. ${ }^{1}$ Their adhesion to surfaces underwater is enabled through the attachment of bubbles entrapped in their setae covered legs. Locomotion, however, is done by applying mechanical forces on the bubbles in order to move, attach and detach them in a controlled manner. In synthetic conditions, however, when a bubble is bound to a surface, it is nearly impossible to maneuver without the use of external stimuli. Thus, actuated wetting and de-wetting of surfaces remain challenges. Here, electrowetting on dielectric (EWOD) is used for the manipulation of bubble-particle complexes on unpatterned surfaces. Bubbles nucleate on catalytic Janus disks adjacent to a hydrophobic surface. By changing the wettability of the surface through electrowetting the bubbles show a variety of reactions, depending on the shape and periodicity of the electrical signal. Time resolved $(\mu \mathrm{s})$ imaging of bubble radial oscillations reveals the possible mechanisms for lateral mobility of bubbles on a surface under electrowetting: bubble instability is induced when electric pulses are carefully adjusted. This instability is adjusted to control the surface bound bubble locomotion and described in terms of the change in surface energy. It is shown that a deterministic force applied normal can lead to a random walk of micrometer-sized bubbles by exploiting the phenomenon of contact angle hysteresis. Finally, bubble use in nature for underwater locomotion and the actuated bubble locomotion presented in this study are compared.
\end{abstract}




\section{INTRODUCTION}

Many plants in nature, especially the lotus leaf, ${ }^{2}$ are famous for their ability to render droplets highly mobile on their surface. ${ }^{3}$ Due to the superhydrophobic nature of these surfaces and minimal pinning ${ }^{4,5}$ a droplet reaching the surface easily rolls off, carrying contaminants on its way. Excessive attempts were made in the last years to mimic such materials that induce droplet mobility on their surface. ${ }^{6-9}$ In addition, the enhancement of droplet locomotion due to coalescence was recently reproduced with synthetic hierarchical structures. ${ }^{10}$ However, an equivalent underwater propulsion mechanism of bubbles at the solid-water interface is yet missing and not without a reason. Surface bound bubbles are highly pinned and particularly challenging to render mobile. A key tool to tune the hydrophobic-hydrophilic interactions of droplets or bubbles with a surface is provided by materials with tunable wettability. Examples of such materials were previously reported using mostly UV irradiation. ${ }^{11-13}$ Calculations show that electric fields can be also potentially used on superhydrophobic graphene surfaces. ${ }^{14}$ However, these reversible transitions between hydrophobic and hydrophilic states are rather slow and involve changes of the surface chemistry. In contrast, electrowetting shows rapid responses to applied electric voltage, modulating the hydrophobicity of dielectric materials. ${ }^{15,16}$ Under applied voltage the hydrophobicity drops, i.e. the contact angle decreases, rendering a hydrophobic surface more hydrophilic. Interestingly, electrowetting is also used for moving droplets and detaching them from hydrophobic surfaces, a fact that broadens the applicability of this method tremendously. ${ }^{17-19}$ It was previously shown that alternating voltage decreases the contact angle hysteresis of droplets. ${ }^{20}$ Another study extended the research on mobility of droplets on superhydrophobic surfaces to hydrophobic surfaces by investigating the shape and periodicity of the applied electric signal. ${ }^{21}$ Electrowetting can be also applied to bubbles, exploiting the same principle of tunable wettability. ${ }^{22}$ It was previously demonstrated that electrowetting of bubbles 
can be used for trapping, transportation and controlled release of particles underwater. ${ }^{23-25}$ This principle was also successfully used for attracting particles remotely. ${ }^{25,26}$ In all these studies the translational motion of the surface bound bubbles is achieved by sequential actuation of adjacent electrodes. $^{23,24,27}$

First we address the main question, whether rendering a non-patterned surface slippery for bubbles in a controlled fashion is possible. In particular, we aim to define the conditions for which radial oscillations of a surface bound bubble, caused by a change in surface wettability, transform into translational motion. The influence of voltage, and consequently change in contact angle, on lateral translational motion is experimentally investigated and discussed in terms of excess in surface energy and contact angle hysteresis. In addition, the influence of the shape and frequency of the applied electrical signal on the dynamics of the bubble motion is studied. The scope of this study is to show for the first time how application of a deterministic force can lead to a stochastic motion of bubbles, which can be used for controlled bubble locomotion, coalescence and possibly detachment from the surface, as was previously shown using electrowetting of droplets.

Here we apply rectangular electric pluses to an electrowettable surface decorated with micometer sized bubbles. By careful tuning of the applied electric pulse and therefore by changing the contact area of the bubble with the surface it is demonstrated, that translational motion can be not only actuated but also regulated. If the periodicity of the actuation pulse is low, there is enough time for relaxation of the bubble without resulting displacement. However, if the periodicity of the actuation pulse is set to a higher frequncy, so that bubble oscillations are continuous, an instability is induced, finally leading to a translational motion. 


\section{RESULTS AND DISCUSSION}

\section{Catalytically active Janus nanodisks}

Catalytically active Janus disks were used for the nucleation of micro bubbles directly on the surface (Figure 1a). The disks are $6 \mu \mathrm{m}$ in diameter and consist of $50 \mathrm{~nm}$ thick Pt and Ni layers (each) on $200 \mathrm{~nm}$ thick silicon nitride (see supporting information). The platinum layer is catalytically active and is used as the bubble nucleation site through the splitting of $\mathrm{H}_{2} \mathrm{O}_{2}$ into $\mathrm{H}_{2} \mathrm{O}$ and $\mathrm{O}_{2}$. As the nanodisks are randomly distributed, the Pt side can be directly in contact with the surface or roughly $250 \mathrm{~nm}$ remote from it. Bubbles nucleating on particles adjacent to a hydrophobic substrate spontaneously adhere to the surface as was previously shown. ${ }^{28}$

\section{Electrowetting of bubbles}

A chamber consisting of upper and bottom indium tin oxide (ITO) electrodes was used for the electrowetting experiments as seen in Figure 1b. A layer of polypropylene (PP) film was put on the bottom electrode. To assure good contact between the PP film and the ITO layer a thin liquid layer of aqueous $\mathrm{KCl}(0.5 \mathrm{M})$ was placed in between. In each experiment, an aqueous droplet containing the Janus disks is placed on the polypropylene film, and $\mathrm{H}_{2} \mathrm{O}_{2}$ (see experimental section) is added in order to nucleate bubbles. The influence of the applied voltage on the bubble is schemed in Figure 1c. The contact area of the bubble with the surface decreases upon applying a voltage, as the contact angle (CA) drops. The disk thickness is on the nanometer scale, and therefore the bubbles easily adhere to the hydrophobic surface. ${ }^{28}$ Below saturation the contact angle $\theta_{\mathrm{V}}$ follows the external voltage $\mathrm{V}$ according to the Young-Lippmann's equation presented in Equation 1. ${ }^{29,30}$ 


$$
\cos \theta_{V}=\cos \theta_{o}+\frac{\varepsilon_{o} \varepsilon_{r} V^{2}}{2 \gamma_{L V} d}=\cos \theta_{o}+\frac{C V^{2}}{2 \gamma_{L V}}=\cos \theta_{o}+\eta
$$

$\gamma_{\mathrm{LV}}$ stands for liquid/vapor interfacial tension, $\theta$ - the apparent contact angle,.. $\varepsilon_{0}-$ vacuum permittivity, $\varepsilon_{\mathrm{r}^{-}}$specific permittivity, $\mathrm{d}$ - thickness of the dielectric layer, C- capacitance and $\eta$ the electric component in electrowetting. The contact angle of the PP film without applied voltage was measured to be $\left\langle\theta_{0}\right\rangle=103^{\circ} \pm 2$. The CA drops to $75^{\circ} \pm 2$ when a voltage of $300 \mathrm{~V}$ is applied (see Figure $\mathrm{S} 1$ in the supporting information). In addition, the dynamic advancing and receding contact angles of a water droplet on the PP film without applied voltage (in a sessile drop configuration) are $\left\langle\theta_{\mathrm{a}}\right\rangle=103^{\circ} \pm 3^{\circ}$ and $\left\langle\theta_{\mathrm{a}}\right\rangle=71^{\circ} \pm 3^{\circ}$ respectively.

\section{Time resolved tracking of bubbles under electrowetting}

First we investigate the transient response of single bubbles to different pulses. In order to understand the influence of the pulse periodicity on the bubble oscillations, a fast camera was used (30,000 fps or $33.3 \mu$ s per frame) together with optical microscopy to resolve the electrowetting behavior in real time and to estimate the distance the bubble travels over the course of one pulse cycle. Using image analysis we tracked bubble diameter and position under the application of pulses of different amplitude and width. We denote Tp and $\mathrm{Tn}$ as the time lengths of the positive and negative square pulses, respectively (see Figure S3 in the supporting information). Ti is the time interval between the positive and negative pulses. In all experiments we consider the symmetric case of $\mathrm{T}=\mathrm{Tp}=\mathrm{Tn}=\mathrm{Ti}$. $\mathrm{P}_{\mathrm{s}}$ is the time between two subsequent pulse cycles and is considerably larger than $\mathrm{T}$ thus, the induced motion in one cycle is independent of the next cycle. Measurements of the bubble diameter and center of mass were extracted from the time resolved optical micrographs as seen in Figure 2. The optical micrographs showing a 
contraction-expansion cycle of a bubble are presented in Figure 2a (full micrograph sequences can be found in the supporting information, Figure S4). Such an image set enables intimate tracking of the bubble diameter and displacement during an electrowetting pulse (Figure 2b). The plots present tracking of bubbles under four kinds of electric pulses: i) $\mathrm{T}=500 \mu \mathrm{s}$, ii) $\mathrm{T}=250$ $\mu \mathrm{s}$, iii) $\mathrm{T}=100 \mu \mathrm{s}$ and iv) $\mathrm{T}=5 \mu \mathrm{s}$, with $\mathrm{V}=300 \mathrm{~V}$ in all cases. The initial diameters of these bubbles are $165,185 \mu \mathrm{m}, 187 \mu \mathrm{m}$ and $214 \mu \mathrm{m}$, respectively. Please note that the bubble diameter was measured using a circle fitting. In some cases there were deviations from a perfect circle due to pinning of the contact line and thus, an error of up to $5 \mu \mathrm{m}$ was measured. The presented diameters are normalized to their initial value in order to compare the bubble contraction in all cases. As seen in Figure 2b (i) the bubble contraction starts with the voltage pulse, however, does not stay constant during the plateau of the pulse. We attribute the lack of a static change in contact angle to the symmetric ITO electrode setup. When the top electrode is replaced by a platinum electrode (which has much greater ability to supply current to the system) measurement of the contact angle against external voltage shows clearly a static response (Figure S1). We note that the rise time of the voltage (from 0 to the maximal voltage) is much longer in the measurement summarized in Figure S1 in the appendix (DC voltage) in comparison to that time in the case of the square pulse. Considering the diffusion constant of the ions in the bulk water in the order of $10^{-5} \mathrm{~cm}^{2} / \mathrm{s}$ and the electrode distance of $1 \mathrm{~mm}$ we find relaxation times for the charge carriers in the bulk medium on the order of micro-seconds (Equation S2 in the supporting information). ${ }^{31}$ We can therefore assume, that during the voltage rise the system of ITO-PP filmITO is not relaxed and the charges necessary for the electrowetting effect, are supplied by a transient polarization current. After reaching the plateau the system is quickly equilibrated to a state, where apparently the PP film is uncharged. In other words the voltage drops elsewhere in the system, likely on the large ITO-electrolyte interface impedance. This means that effectively 
the derivative of the applied pulse is the stimulus to the bubble. While it is outside the scope of this work to further study this effect, we focus on the instabilities of the bubbles induced by these transient processes. In Figure $2 \mathrm{~b}$ (iv) the pulse with the shortest period $\mathrm{T}=5 \mu \mathrm{s}$ is shown. Here the bubble is limited by its dynamic response to the pulse as it can be seen by negligible contraction. When the pulse width is increased, we find that the bubble contraction-expansion cycles relax with a characteristic time of approximately $\mathrm{T}_{\mathrm{r}}=250 \mu \mathrm{s}$, independent of the pulse width. Hence, this is considered as the characteristic time of the bubble radial oscillations. It is now interesting to explore the effect of the pulse period $\mathrm{T}$ on the relaxation time. While only small displacement during the contraction cycle is recorded for both $\mathrm{T}=500 \mu \mathrm{s}>\mathrm{T}_{\mathrm{r}}$, and $\mathrm{T}=5 \mu \mathrm{s}, 100 \mu \mathrm{s}<\mathrm{T}_{\mathrm{r}}$ a maximal displacement as large as $14 \mu \mathrm{m}$ is recorded for $\mathrm{T}=250 \mu \mathrm{s} \approx \mathrm{T}_{\mathrm{r}}$. Further, we like to point out that displacement of the bubble center of mass during the oscillation differs from the final displacement at the end of the cycle. It is also interesting to point out that changes in polarity do not affect the bubble response to electrowetting, as seen in the plots in Figure 2b. Considering that the CA of the surface in the absence of applied voltage is $\left\langle\theta_{0}\right\rangle=103^{\circ} \pm 2$ and the contraction of the bubble to $75 \%$ of its original diameter in cases (i) and (ii), one can estimate the final CA of the surface from the geometry of the bubble using the illustration in Figure 1c. According to these geometrical considerations, the final diameter of the bubble corresponds to a $\mathrm{CA}$ of $\sim 77^{\circ}$, in consistence with the independent measurements of the electrowetting curve (see Figure S1 in the supporting information) and in agreement with the argument above, that we witness a transient but complete electrowetting response during the fast voltage rise. Contact angles corresponding to contractions down to 80,85 and $90 \%$ of the bubbles original diameter were calculated as well and resulted in $\mathrm{CA} \sim 83,88$ and $92^{\circ}$ respectively. To further study the bubble displacement we measured the macroscopic path traveled by the bubbles over many pulse cycles. 
Bubble dance: regulating bubble mobility by tuning electrowetting periodicity

Now we applied a series of 500 pulse cycles of duration T. Consistent with the previous single pulse experiments we find that tuning the pulse width influences the macroscopic path the bubbles travel. Tracking of bubble motion under electrowetting with varying parameters is presented in Figure 3. The average travelling distance was calculated along 500 pulse cycles, and the statistics is based on analyzing tracks of at least 15 bubbles per configuration. Bubble catalytic growth is much slower than the time scale of the applied voltage, and therefore the bubble volume is presumed to be constant during the experiment. Detached bubbles accumulate at the top of the chamber and exhibit neither oscillations nor translational motion. Thus, the bubbles are not influenced by Maxwell stresses caused by the transient electric field directly but rather by the change in the surface wettability, as formulated in the Young-Lippmann's equation. Figure 3a presents optical micrographs extracted from videos recorded at $2 \mathrm{fps}$ (or $500 \mathrm{~ms}$ per frame). One can clearly see the differences between the mobility of the bubbles for different pulse settings. For very short electrowetting pulses $(\mathrm{T}=5,10 \mu \mathrm{s})$ the bubbles do not perform full oscillations, and therefore their displacement is hindered, while continuous full contractionexpansion cycles result in large displacements as seen in the latter cases ( $\mathrm{T}=75,150$ and $250 \mu \mathrm{s})$. A careful adjustment of the oscillations is the key point for tuning the bubble mobility. The influence of the pulse periodicity on the bubble displacement is summarized in Figure 3b for pulses of $\mathrm{T}=5,15,75,150,250 \mu$ s with $\mathrm{P}_{\mathrm{s}}=20 \mathrm{~ms}$. In these experiments the voltage amplitude was set to $300 \mathrm{~V}$. As was previously discussed, a full contraction cycle lasts roughly $250 \mu \mathrm{s}$ (Figure 2b). When adjusting the periodicity of the contractions, so that a bubble is under continuous transitional state of changing its contact area with the surface, bubbles start showing significant enhancement in translational motion. The maximal traveling path over 500 pulses can reach almost $3000 \mu \mathrm{m}$, one to two orders of magnitude higher than the bubble dimensions. It is 
inferred from Figure 2 that the displacement of bubbles takes place only during the bubble contraction-expansion cycle. Therefore, the value of $\mathrm{P}_{\mathrm{s}}$ (the time interval between the pulses) does not influence the magnitude of the traveling path.

\section{Bubble random walk underwater}

Now we link the traveling path along one pulse cycle, as seen in Figure 2, to the macro displacement, as seen in Figure 3a. We consider the model of a random walk with the traveled end-to-end distance $R$ and displacement $\mathrm{r}$, for $N$ steps with a displacement, or jump, of $a$ (Equation 2). ${ }^{32}$

$R=\sqrt{\overline{r^{2}}}=a \sqrt{N}$

Over the course of $500 \mathrm{~ms}$ (video resolution) many oscillation and displacement cycles take place $(\mathrm{T}=250 \mu \mathrm{s})$. In the limit of continuity considered here, the squared distance from the origin $r^{2}$ in a random walk model follows the probability distribution in Equation 3 . $^{33}$

$P_{d}\left(r^{2}, t\right)=1-\exp \left(r^{2} / r(t)_{0}^{2}\right)$

$\mathrm{P}_{\mathrm{d}}$ is the probability to find a bubble a distance equal to or smaller than $r^{2}$ after a number of steps or time t. From the acquired movies we could track and extract the bubble path and calculate the corresponding probability distribution. We counted the number of bubbles, which were within a circle of radius $r$ from their starting point for a fixed time $t$. To normalize, the distribution is scaled by the total number of points. A total of 18 bubbles over the time course of 10 seconds were considered. The calculated probability distribution along with the fit to Equation 3 is shown 
in Figure 3d (note the logarithmic scale). We obtain remarkable agreement for small to intermediate distances. We conclude that the bubbles indeed follow a random walk. In larger distances bubbles coalesce and thus cannot be considered. We would like to note that a wide range of bubble sizes (approximately $80-500 \mu \mathrm{m}$ in diameter and $240 \mu \mathrm{m}$ in average) was considered in the analysis and yet we obtain a good fit $\left(\mathrm{R}^{2}=0.982\right)$ to a one-population model. We conclude that for the bubble sizes considered the jump distance $a$ does not depend strongly on the size of the bubble. This can be understood from an energy balance for a single bubble displacement shown in Equation 4.

$\int d \vec{s} \vec{F}_{\text {drag }}=\pi r_{b}^{2} C / 2 V^{2}$

Where $F_{\text {drag }}$ is the drag force, $r_{b}$ is the radius of the bubble, $C$ is the PP film capacitance per unit area, which can be deduced from Equation 1. For the condition in which the kinetic movement dominates over relaxation by oscillations and under the assumption of a steady state during small linear displacements $a$ we find (5) $a \sim \frac{r_{b}^{2} C}{F_{d r a g}} V^{2}$. Neglecting contact line effects, $F_{\text {drag }}$ will be dominated by the surface friction and the fluid drag force and hence will roughly scale as $r^{2}$. Hence the distance of the displacement per jump will not depend on the bubble size. This confirms the previous analysis using Equation 3. Finally we test the relation of the displacement to squared applied voltage predicted as $r \sim V^{2}$ (Equations 2 and 5). We find good agreement between this simple theory and data for the intermediate set of voltages (Figure 3c). For voltages of $100 \mathrm{~V}$ and below bubble displacement is minor. In this range, the change in CA is roughly $10^{\circ}$. However, when the voltage exceeds $200 \mathrm{~V}$ the CA drops to approximately $75^{\circ}$, and displacement is observed. Although saturation of the contact angle was measured above $200 \mathrm{~V}$, it can be 
inferred from the plot, that displacement continues to increase from roughly $200 \mu \mathrm{m}$ to more than $500 \mu \mathrm{m}$ (mean values) with voltage in the range of $200-300 \mathrm{~V}$. It is important to remember that the electric component in the Young-Lippmann's equation, which represents the electric contribution in terms of electric force (or energy) acting on the contact line, scales with the square voltage. Hence, it is possible, that the electric force continues to increase in this voltage range and can further induce displacement while the CA remains saturated.

\section{System model for bubble translational motion}

In order to estimate the contribution of the incomplete relaxation of the bubble to its translational motion, the energy of a bubble during a contraction cycle is considered. This energy was calculated using the terms shown in Figure 4. Two states are taken into account: a) the initial/final states, corresponding to $\mathrm{CA}=103^{\circ}$ and $\mathrm{b}$ ) fully contracted bubble under voltage of $300 \mathrm{~V}$, corresponding to $\mathrm{CA}=75^{\circ}$. Before a bubble is subjected to a voltage in an electrowetting experiment its initial surface energy $\mathrm{E}_{\mathrm{i}}$ is given by:

$E_{i}=A_{i} \gamma_{S V}+S_{i} \gamma_{L V}$

Under applied voltage the surface energy $\mathrm{E}_{\mathrm{f}}$ is given by:

$E_{f}=A_{f} \gamma_{S V}+\left(A_{i}-A_{f}\right) \gamma_{S L}+S_{f} \gamma_{L V}$

Finally, when the voltage is no longer applied, the bubble energy is described again by $\mathrm{E}_{\mathrm{i}}$. As was previously discussed, translational motion of the bubble is observed already when contraction starts to take place. Considering the time resolution of $33.3 \mu$ s per frame, it is possible to estimate the velocity of a bubble during a pulse. Analyzing the bubble displacement based on 
the plot in Figure $2 \mathrm{~b}$ (ii) (for a pulse of $\mathrm{T}=250 \mu \mathrm{s}$ ), the velocity of the bubble, when contraction starts, is approximately one hundred nanometers. However, when the bubble diameter does not fully recover before another contraction takes place $(\mathrm{t} \sim 750 \mu$ s in Figure $2 \mathrm{~b}$ (ii)) an additional mobility is induced. In this case, displacement of roughly half a micron is recorded- roughly five times the displacement of the in-place oscillations.

According to energy calculations, the maximal energy difference between the two states is roughly $10^{-10} \mathrm{~J}$ for a bubble with an initial diameter of $165 \mu \mathrm{m}$. The values used for the calculations are $\gamma_{\mathrm{LV}}=72 \mathrm{mN} / \mathrm{m}$ and $\gamma_{\mathrm{SV}}=30 \mathrm{mN} / \mathrm{m}$ for the polypropylene film. ${ }^{34}$ Clearly this is the upper limit in energy difference between the two states. The Reynold's number in the system is approximately 10 (see supporting information), thus this excess of surface energy can be related to kinetic energy. Nevertheless, the actual fraction of energy potentially transformed into kinetic energy is much smaller, considering drag forces and heating. Additionally, the contact line of the bubble with the surface is subjected to pinning, decreasing its mobility. An example of an incomplete radial oscillation can be seen in Figure $2 b$ (ii). Such an oscillation corresponds in theory to an energy difference of $0.5 \cdot 10^{-10} \mathrm{~J}$. In terms of kinetic energy, this corresponds to a velocity of $7 \mu \mathrm{m} / \mu \mathrm{s}$. From the plot it can be inferred, that the bubble displacement at $\mathrm{t} \sim 750 \mu \mathrm{s}$ is roughly $15 \mu \mathrm{m}$ (measured between two consecutive frames). Therefore, the velocity of the bubble is approximately $0.5 \mu \mathrm{m} / \mu \mathrm{s}$. As expected, the real velocity of the bubble is much smaller than the theoretical value considering that the bubble needs to overcome the surface adhesion force, local pinning and shear forces acting on the contact line during the radial oscillation. ${ }^{35,36}$

At last we want to comment on the origin of the directional movement. The displacement of the bubble has to be driven by a gradient in surface energy, as the applied field is normal to the surface. In principle, a displacement of a bubble is possible, if the change in the contact angle of the substrate, on which the bubble is situated, exceeds the range of the contact angle hysteresis. In 
other words, the contact angle hysteresis can be treated as the solid-liquid adhesive force, and overcoming this adhesion, it is possible to actuate translational motion. ${ }^{24,37}$ When droplets are discussed, two competitive mechanisms, that can describe the behavior of metastable states on a smooth heterogeneous surface, were previously suggested. ${ }^{38}$ In the first, a droplet tends to remain in its asymmetric state, while energy is being reduced, by reshaping for example. In the second mechanism, the meta-stability is a driving force for the droplet to move toward a position, in which its energy is reduced. In the case of bubbles, several parallel mechanisms can be responsible for translational motion. Local pinning due to the surface roughness of the dielectric substrate or the presence of the nanodisks cause the bubble center to dislocate, as seen in Figure $\mathrm{S} 5$ in the supporting information. For the same reason, the electrical component acting parallel to the surface is not symmetric as well. Finally, if the bubble lacks sufficient time to reorient, following the instantaneous change in contact angle, an additional instability is introduced. The energy of the bubble in this metastable state can be reduced, if the bubble changes its position. These mechanisms are acting in parallel, however, only the last one can explain the striking differences in bubble response to pulsed voltage.

Comparison between bubble use in nature and artificial actuation of bubbles for underwater locomotion

Insects, specifically terrestrial beetles, capable of walking underwater by using entrapped bubbles in their legs, were first reported by Hosoda et al. ${ }^{1}$ The mechanism of locomotion on solid surfaces underwater is a combination of hydrophobic interactions, enforced attachment and detachment of bubbles from the surface and capillary forces. Traction forces generated by the leaf beetle on hydrophobic surfaces range between 3 and $8 \mathrm{mN}$. This translates to work in the range of a few dozen $\mathrm{nJ}$ for distances in the micrometer scale. Considering Equation 6 and 7 in this study, the 
work performed by electrowetting on a surface anchored bubble of $165 \mu \mathrm{m}$ in diameter is approximately $0.1 \mathrm{~nJ}$, smaller than what observed in nature. However, this is logic considering some fundamental differences between the two cases. First, the bubbles used by insects are entrapped in a setal structure that stabilizes them in comparison to the bubbles in this study, which are anchored to the surface only. As a result, these two systems differ geometrically. Additionally, the contact area of the beetle tarsomere with the surface is roughly $300 \mu \mathrm{m}$ in width and $150 \mu \mathrm{m}$ in length, ${ }^{39}$ while we consider a smaller round bubble of $165 \mu \mathrm{m}$ in diameter for the energy calculations. Finally, it is important to remember that although traction forces were previously measured, the beetles actually use reversible attachment and detachment from the surface using air bubbles in order to perform underwater locomotion.

\section{Actuated functionalities induced by mobility}

We have established how and under which conditions bubbles can be actuated to move across an unpatterned surface. Now we show that bubble mobility on the surface can be used to fulfill a variety of tasks. ${ }^{24,40-42}$ A crucial problem when using micro-fluidic devices is clogging by bubbles or by contaminations. Here it is suggested that actuated motion of bubbles can be used to clean and unblock micro-channels. Figure 5 shows how mobility of bubbles on the surface could be beneficial to perform a variety of functions at the solid/water interface. Mobility is demonstrated to be useful for: coalescence, trapping, carrying cargo and bubble transport. Coalescence of adjacent bubbles is shown in Figure 5a. This depends on the mobility and distribution of the bubbles. According to Figure 4 coalescence of micro bubbles within the range of millimeters is possible upon adjustment of the electrowetting pulse. This range is attractive for microfluidic devices, in which the channels are usually smaller than this order of magnitude. In addition, trapping of bubbles is possible, if traps are in the vicinity of mobile bubbles. In Figure 
$\mathbf{5 b}$ it is shown, that a bubble is trapped in a circularly shaped island, which is not subjected to electrowetting. The electrowetting setup used in this study uses a thin conductive layer between the electrode and the dielectric PP layer. In an area, where the conductive aqueous medium is absent, bubbles are no longer subjected to electrowetting and thus, once a bubble reaches a trap, it is fixed there. Upon locating these traps in known positions one can guide and predetermine the final position of the microbubbles. As more bubbles come close to this trap they coalesce to form bigger bubbles, which eventually have to detach under buoyancy forces. In Figure 5c one can see a contamination carried by a bubble along its path. The dark spheres are polystyrene microparticles $(16 \mu \mathrm{m})$. As seen in the image sequence, and in accordance with previous studies, the small particles are not being trapped in contrary to the larger contamination. Finally, adjacent bubbles are able to attract each other due to secondary Bjerknes forces. ${ }^{43}$ In Figure 5d one can see a bubble pair traveling together due to Bjerknes attractive forces.

\section{CONCLUSIONS}

In this study, underwater locomotion of surface anchored bubbles is actuated by electrowetting. It is shown that by proper tuning of voltage periodicity a bubble instability can be induced, promoting translational motion of up to two orders of magnitude higher than the bubble size. Time resolved $(\mu \mathrm{s})$ imaging suggests that there is a threshold above which translational motion of surface anchored bubbles is preferred over radial oscillations. The translational motion fits a random walk model so that the hydrophobic surface becomes "slippery" for bubbles. The voltage in terms of amplitude and frequency dictates the degree of mobility of the bubbles and is a key tool to regulate their motion. The excess of interfacial energy of a bubble in the course of one contraction-expansion cycle is calculated in order to estimate its contribution to the translational movement on the surface. The calculated energies are compared with previous studies dealing 
with underwater locomotion via bubble use as performed by several insects in nature. Finally, additional outcomes of bubble mobility on the surface are demonstrated: actuated coalescence, trapping and cargo transport. Actuated motion of bubbles on hydrophobic surfaces could be of great interest for applications in the field of underwater adhesion, underwater cleaning mechanisms, microfluidics and lab on chip devices in wet environments. An outlook of this work envisions directing motion of bubbles inside micro-fluidic channels and actuated motion of bubbles along wettability gradients to induce directionality to the motion, crucial to the applicability of these findings. Another interesting aspect is the dynamic response of bubbles to electrowetting using electric waves of different shapes and surfaces with hierarchical structures. ${ }^{44,45}$ Such surfaces might be suitable for inducing the transition between lateral mobility and controlled detachment of bubbles from the surface.$^{46}$ Finally, an interesting aspect yet to be explored is the response of bubbles of smaller dimensions (nanobubbles) to electrowetting on hydrophobic surfaces. ${ }^{47,48}$

\section{EXPERIMENTAL}

Chemicals: polypropylene film, $4 \mu \mathrm{m}$ thick was provided by Goodfellows $\mathrm{GmbH} . \mathrm{H}_{2} \mathrm{O}_{2}(30 \%)$ and $\mathrm{KCl}$ were provided by Sigma-Aldrich. Polystyrene beads (16 $\mu \mathrm{m}$ in diameter) were provided by Microparticles GmbH.

Instrumentation: A Zeiss (axiovert 135TV, Germany) microscope was used for time resolved optical imaging. Time resolved imaging was done by a fast digital camera HG-100K (Redlake Inc., San Diego, CA) .A Leica (M165C) microscope was used for optical imaging. Images were analyzed by Image J, and Matlab R2014a was used for calculations.

Experimental: in each experiment $10 \mu \mathrm{L} \mathrm{KCl}(0.5 \mathrm{M})$ was spread between the ITO covered glass and the PP film. A droplet containing $20 \mu \mathrm{L}$ nanodisk suspension, $5 \mu \mathrm{L} \mathrm{KCl}(0.5 \mathrm{M})$ and $20 \mu \mathrm{L}$ 
distilled water was spread on the PP film followed by the addition of $5 \mu \mathrm{L} \mathrm{H}_{2} \mathrm{O}_{2}(20 \%$ in distilled water). The conductivity of $10 \% \mathrm{v} / \mathrm{v} \mathrm{KCl}(0.5 \mathrm{M})$ in distilled water was measured to be 5.23 $\mathrm{mS} / \mathrm{cm}$. 


\section{FIGURES}

a)

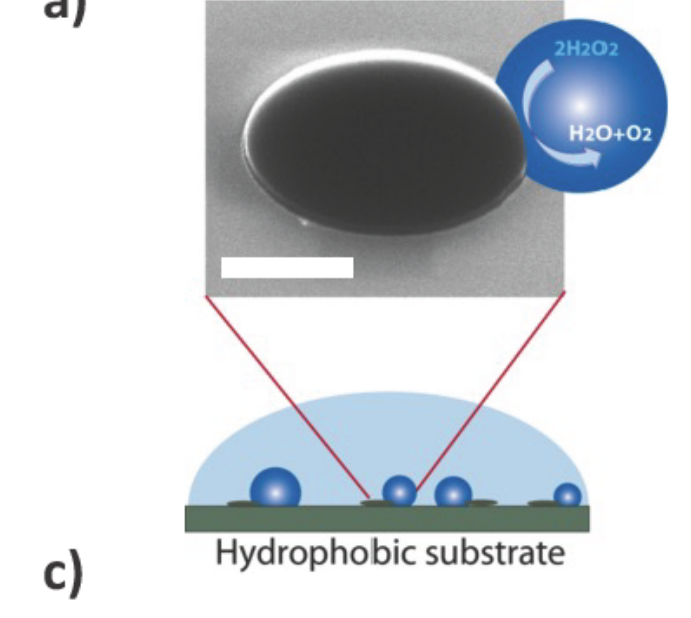

b)

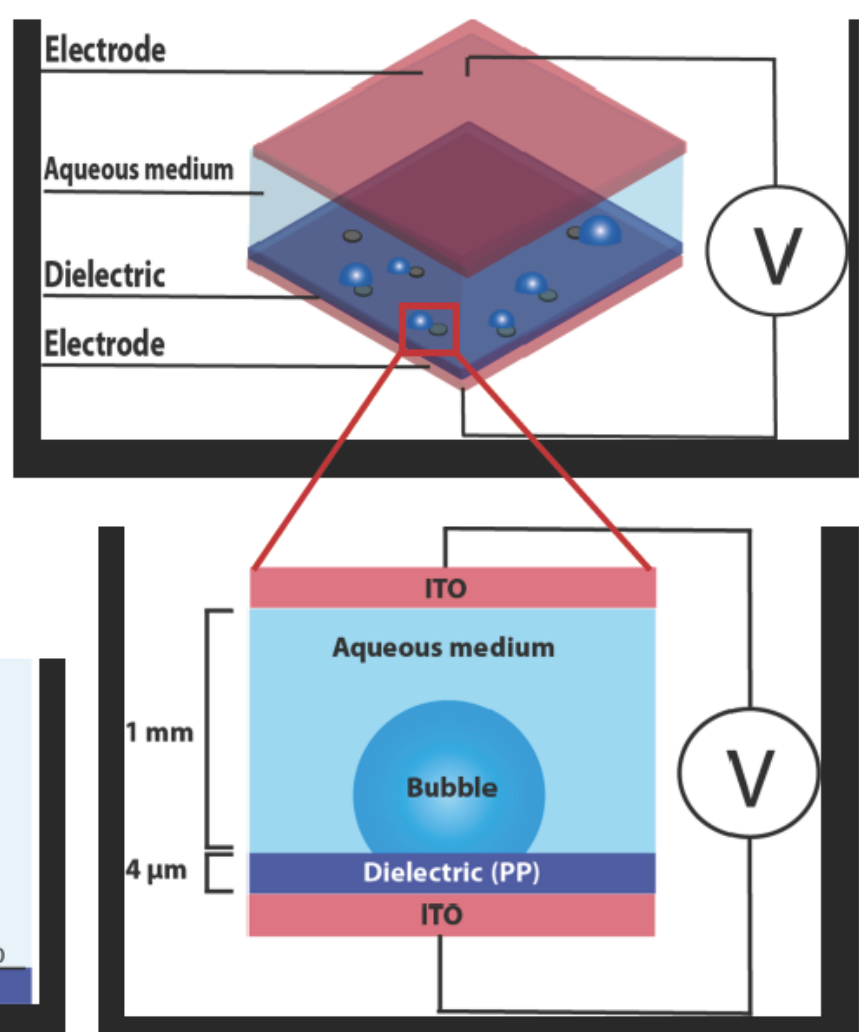

Figure 1. a) Illustration of Janus disks dispersed on the solid-water interface, while bubbles nucleate on the catalytically active Pt side of the particles (inset: A scanning electron micrograph of a single Janus particle). Scale bar corresponds to $3 \mu \mathrm{m}$. b) Illustration of the chamber designed for this study: bottom electrode (ITO), dielectric polypropylene layer and an upper electrode (ITO). The Janus disk-bubble complexes are distributed on the polypropylene layer. c) Illustration showing the influence of a voltage on the wettability of the surface and consequently on the bubble contact area with the surface. The geometry is defined by $\mathrm{h}$ - the bubble height, $\mathrm{r}_{\mathrm{b}}{ }^{-}$ radius of curvature, a- the radius of the contact area with the surface and $\theta$ - the contact angle of the liquid with the surface. By application of voltage to the external electrodes the contact area of the bubble with the surface decreases. 

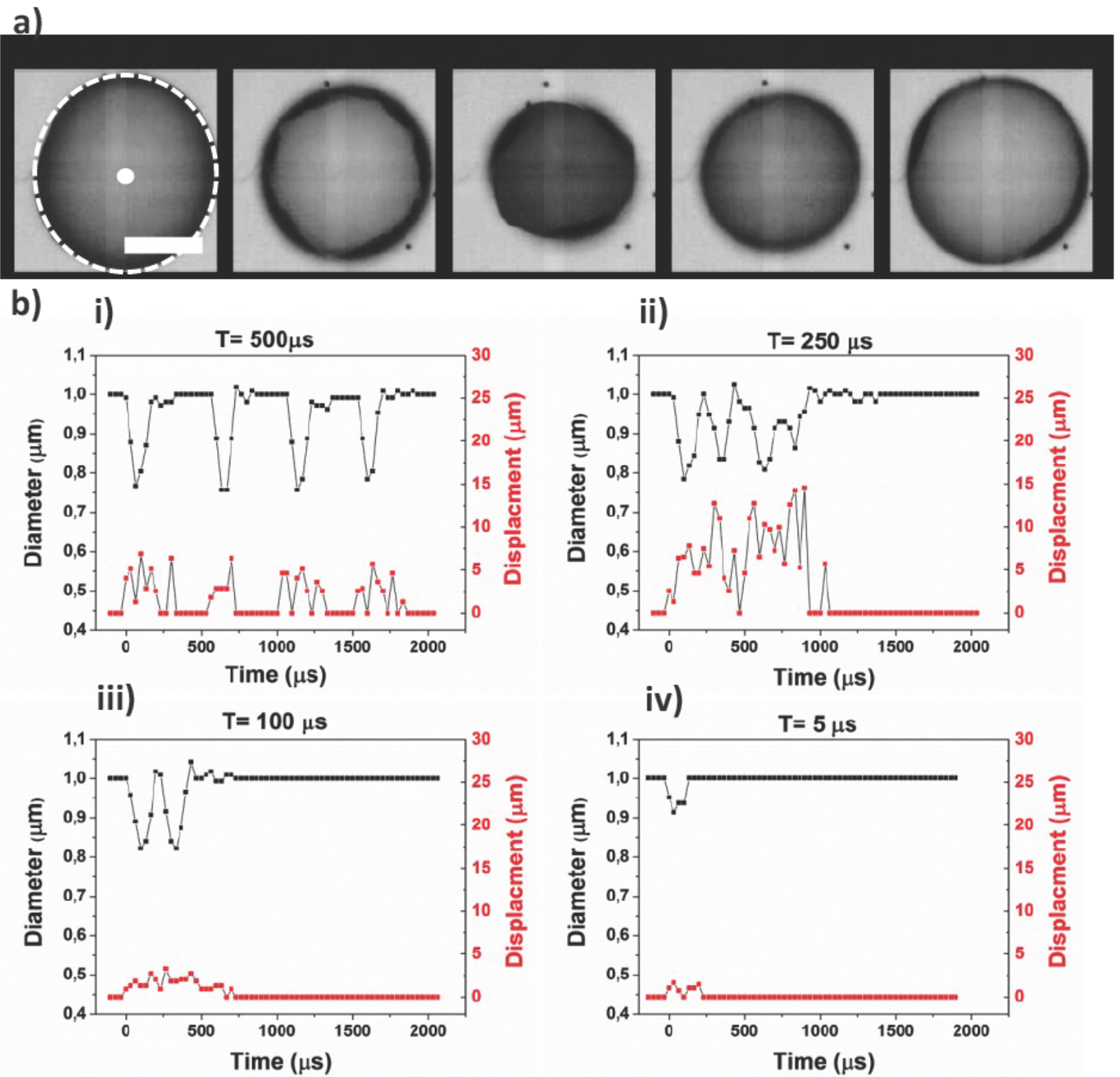

Figure 2. a) Time resolved optical microscopy of bubble radial oscillations on the surface. a) Optical micrographs showing a bubble contraction-expansion cycle as a result of electrowetting for $\mathrm{T}=250 \mu \mathrm{s}$. The bubble diameter and center were tracked based on such image sets. Scale bar corresponds to $100 \mu \mathrm{m}$. b) Plots showing the diameter and displacement of bubbles subjected to four kinds of pulses: i) $\mathrm{T}=500 \mu \mathrm{s}$, ii) $\mathrm{T}=250 \mu \mathrm{s}$, iii) $\mathrm{T}=100 \mu \mathrm{s}$ and iv) $\mathrm{T}=5 \mu \mathrm{s}$. 
a)
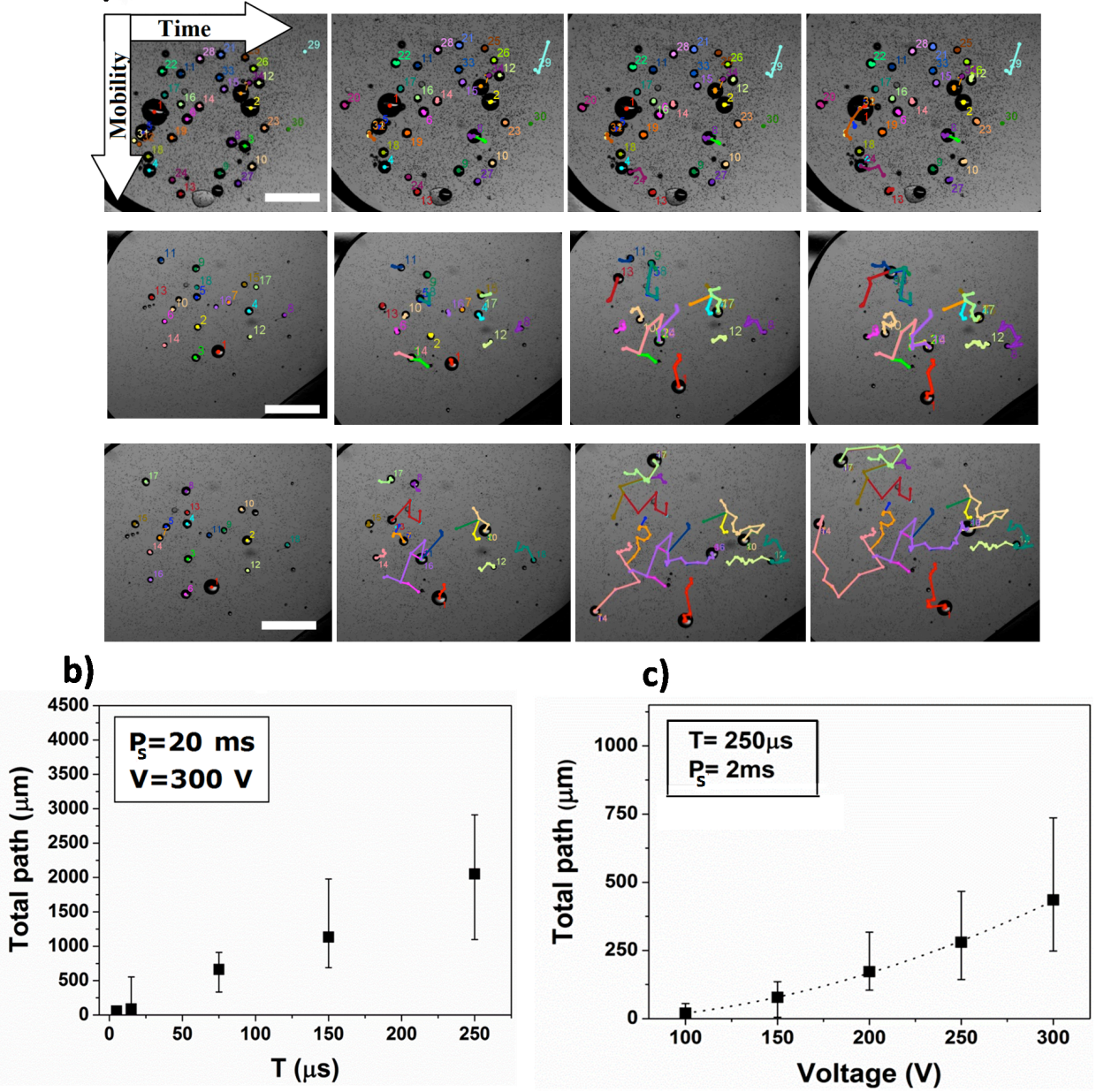

d)
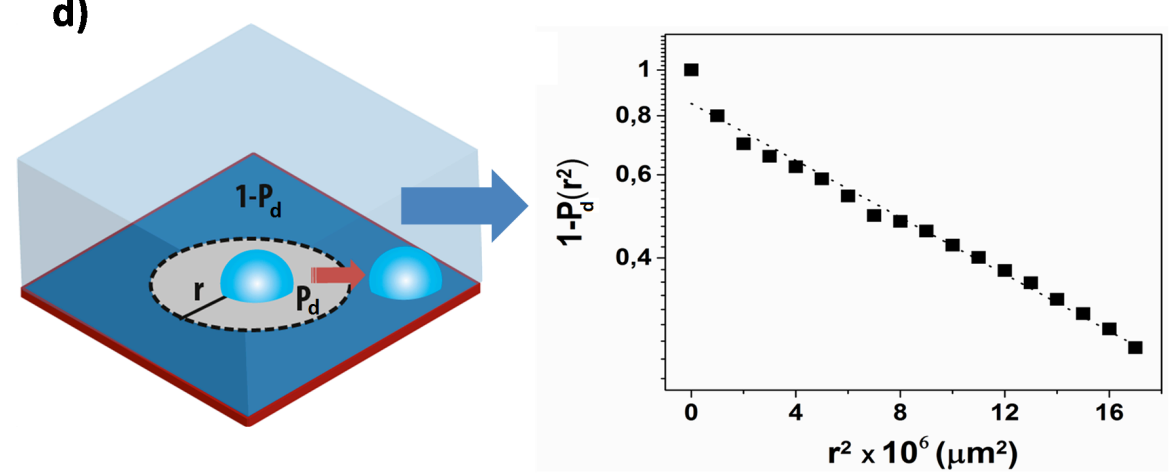

Figure 3. a) Bubble translational motion depending on signal periodicity and voltage. a) Optical micrographs showing the displacement of bubbles tracked along 5500 successive pulse cycles. Each bubble is marked with a different color. The tracks were used to calculate the mean traveling path of bubbles under different conditions. Scale bar corresponds to $1 \mathrm{~mm}$. Plots presenting statistics of the total traveling path of bubbles, b) depending on the oscillation periodicity for pulses with $\mathrm{T}=5,15,75,150$ and $250 \mu \mathrm{s}$ and $\mathrm{P}_{\mathrm{s}}=20 \mathrm{~ms}$ and c) depending on the voltage for a pulse of $\mathrm{T}=250 \mu \mathrm{s}$ and $\mathrm{P}=2 \mathrm{~ms}$. For the statistics 500 successive pulse cycles were considered. d) Probability distribution $1-\mathrm{P}_{\mathrm{d}}\left(\mathrm{r}^{2}\right)$ with fit to equation 2 . 


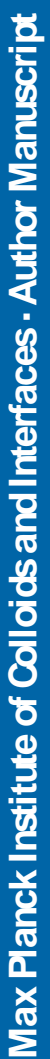

$\mathbf{V} \neq \mathbf{0}$

Energy

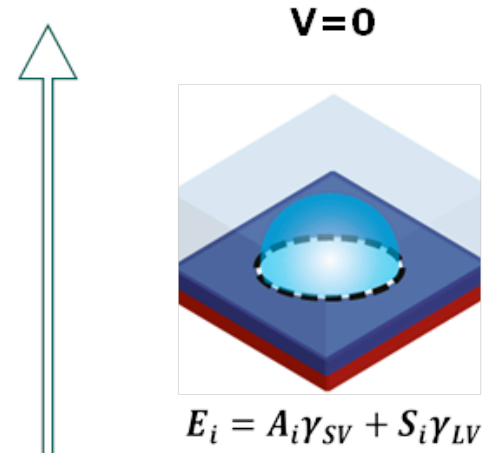

$$
V=0
$$

$\nabla$

$$
E_{f}=A_{f} \gamma_{S V}+\left(A_{i}-A_{f}\right) \gamma_{S L}+S_{f} \gamma_{L V}
$$

$E_{i}=A_{i} \gamma_{S V}+S_{i} \gamma_{L V}$

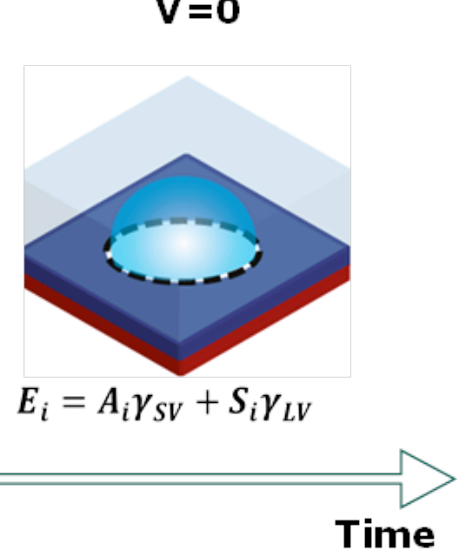

Figure 4. Energy of a bubble during a contraction-expansion cycle. Illustration presenting a bubble attached to a surface of different contact angles and their corresponding energies (bottom). a) Initial state on a hydrophobic substrate $\left(\mathrm{CA}>90^{\circ}\right)$. b) Final state on a hydrophilic substrate $\left(\mathrm{CA}<90^{\circ}\right)$, corresponding to a substrate under an applied voltage. The volume of the bubble remains constant.

22 


\section{Coalescence}

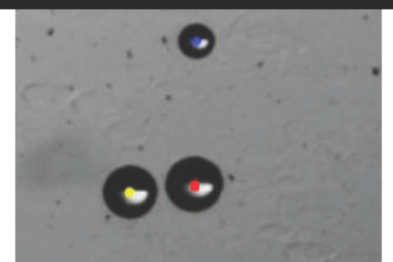

b) $\quad t=0 \mathrm{~s}$

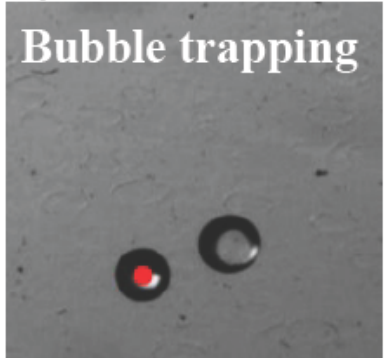

c) $\quad t=0 \mathrm{~s}$

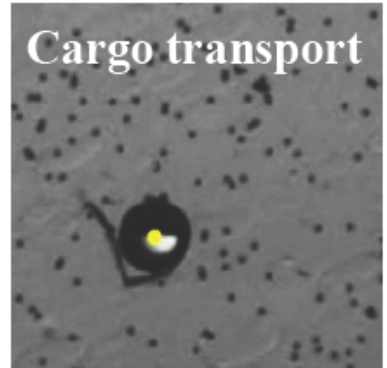

d) $\mathbf{t}=\mathbf{0 ~ s}$

Bubble transport

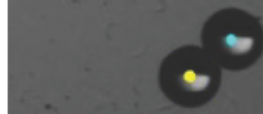

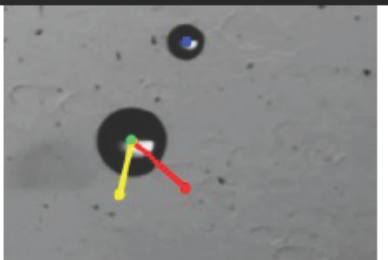

$1 \mathrm{~s}$

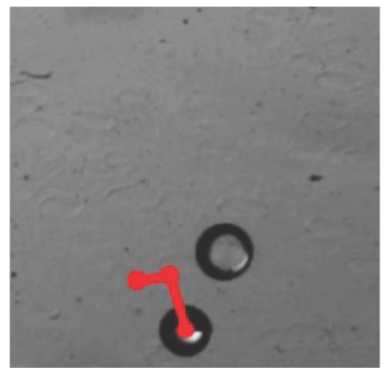

$0.5 \mathrm{~s}$

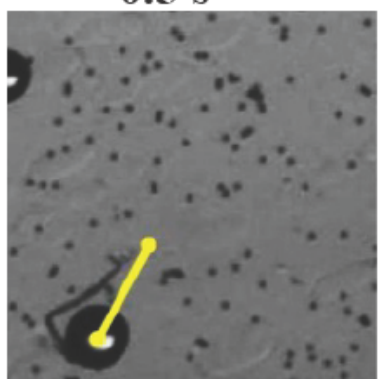

0.5

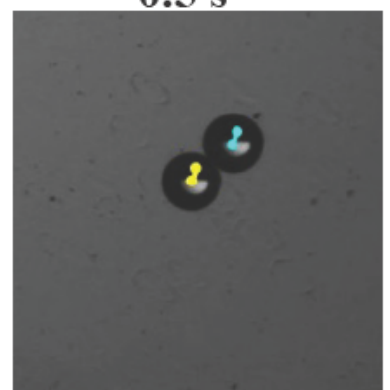

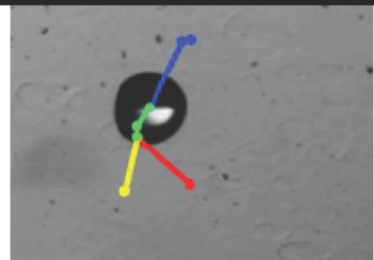

$2.5 \mathrm{~s}$

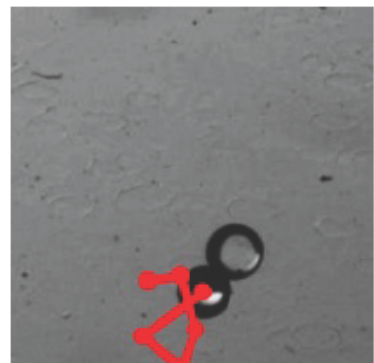

$3 \mathrm{~s}$

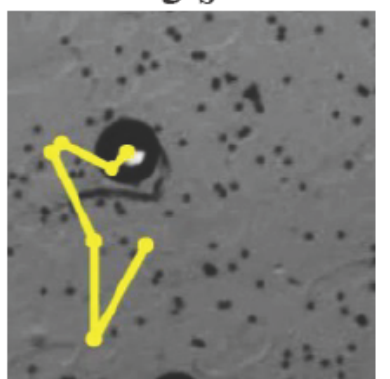

$3 \mathrm{~s}$

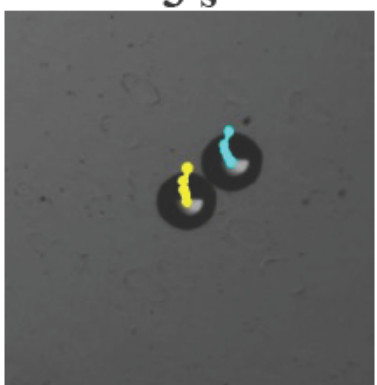

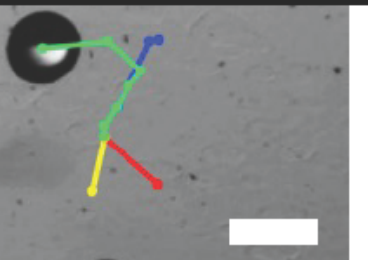

$3 \mathrm{~s}$

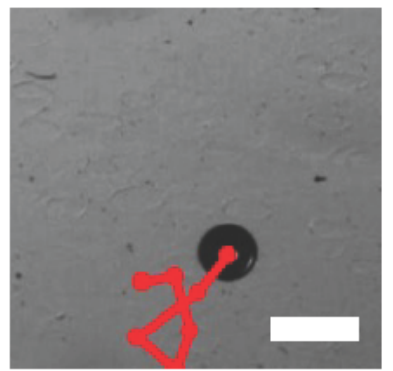

$4 s$

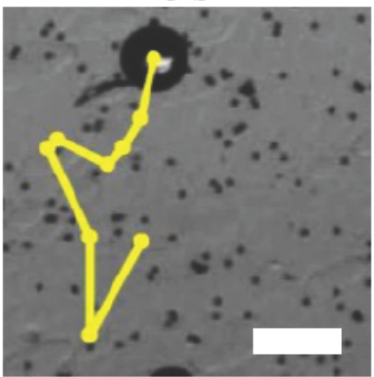

$4 \mathrm{~s}$

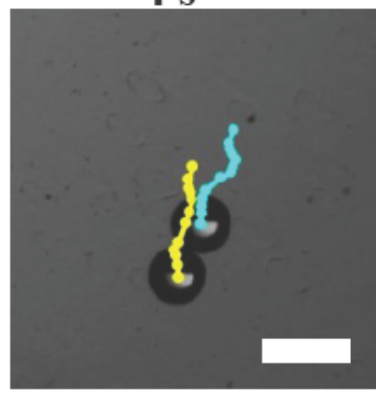

Figure 5. Functions induced by bubble translational motion: a) coalescence, b) trapping, c) cargo transport and d) bubble transport. Scale bar corresponds to $200 \mu \mathrm{m}$. 
Supporting Information. Supplementary videos and supporting information is available free of charge via the Internet at http://pubs.acs.org.

\section{AUTHOR INFORMATION}

\section{Corresponding Author}

* batel.pinchasik@mpikg.mpg.de

\section{REFERENCES}

(1) Hosoda, N.; Gorb, S. N. Underwater Locomotion in a Terrestrial Beetle: Combination of Surface de-Wetting and Capillary Forces. Proc. Biol. Sci. 2012, 279 (1745), 42364242.

(2) Barthlott, W.; Neinhuis, C. Purity of the Sacred Lotus, or Escape from Contamination in Biological Surfaces. Planta 1997, 202 (1), 1-8.

(3) Marmur, A. The Lotus Effect: Superhydrophobicity and Metastability. Langmuir 2004, 20 (9), 3517-3519.

(4) Ling, W. Y. L.; Lu, G.; Ng, T. W. Increased Stability and Size of a Bubble on a Superhydrophobic Surface. Langmuir 2011, 27 (7), 3233-3237.

(5) Wong, W. S. Y.; Nasiri, N.; Liu, G.; Rumsey-Hill, N.; Craig, V. S. J.; Nisbet, D. R.; Tricoli, A. Flexible Transparent Hierarchical Nanomesh for Rose Petal-Like Droplet Manipulation and Lossless Transfer. Adv. Mater. Interfaces 2015, 2, 1500071.

(6) Feng, L.; Li, S.; Li, Y.; Li, H.; Zhang, L.; Zhai, J.; Song, Y.; Liu, B.; Jiang, L.; Zhu, D. Super-Hydrophobic Surfaces: From Natural to Artificial. Adv. Mater. 2002, 14 (24), $1857-1860$. 
(7) Jiang, L.; Zhao, Y.; Zhai, J. A Lotus-Leaf-like Superhydrophobic Surface: A Porous Microsphere/Nanofiber Composite Film Prepared by Electrohydrodynamics. Angew.

Chem. 2004, $116(33), 4438-4441$.

(8) Zhang, L.; Wu, J.; Wang, Y.; Long, Y.; Zhao, N.; Xu, J. Combination of

Bioinspiration: A General Route to Superhydrophobic Particles. J. Am. Chem. Soc.

2012, 134, 9879-9881.

(9) Yu, Y.; Chen, H.; Liu, Y.; Craig, V. S. J.; Wang, C.; Li, L. H.; Chen, Y.

Superhydrophobic and Superoleophilic Porous Boron Nitride

Nanosheet/Polyvinylidene Fluoride Composite Material for Oil-Polluted Water

Cleanup. Adv. Mater. Interfaces 2015, 2, 1400267/1-1400267/10.

(10) Rykaczewski, K.; Paxson, A. T.; Anand, S.; Chen, X.; Wang, Z.; Varanasi, K. K.

Multimode Multidrop Serial Coalescence Effects during Condensation on Hierarchical Superhydrophobic Surfaces. Langmuir 2012, 29 (3), 881-891.

(11) Li, H.; Zheng, M.; Liu, S.; Ma, L.; Zhu, C.; Xiong, Z. Reversible Surface Wettability Transition between Superhydrophobicity and Superhydrophilicity on Hierarchical Micro/nanostructure ZnO Mesh Films. Surf. Coat. Technol. 2013, 224, 88-92.

(12) Lim, H. S.; Kwak, D.; Lee, D. Y.; Lee, S. G.; Cho, K. UV-Driven Reversible Switching of a Roselike Vanadium Oxide Film between Superhydrophobicity and Superhydrophilicity. J. Am. Chem. Soc. 2007, 129 (14), 4128-4129.

(13) Xu, Z.; Ao, Z.; Chu, D.; Younis, A.; Li, C. M.; Li, S. Reversible Hydrophobic to Hydrophilic Transition in Graphene via Water Splitting Induced by UV Irradiation. Sci Rep 2014, 4 .

(14) Jiang, Q. G.; Ao, Z. M.; Chu, D. W.; Jiang, Q. Reversible Transition of Graphene from Hydrophobic to Hydrophilic in the Presence of an Electric Field. J. Phys. Chem. C $2012116(36) 19321-19326$ 
(15) Klarman, D.; Andelman, D.; Urbakh, M. A Model of Electrowetting, Reversed Electrowetting, and Contact Angle Saturation. Langmuir 2011, 27, 6031-6041.

(16) Chen, L.; Bonaccurso, E. Electrowetting - From Statics to Dynamics. Adv. Colloid Interface Sci. 2014, 210, 2-12.

(17) Jun Lee, S.; Lee, S.; Hyoung Kang, K. Droplet Jumping by Electrowetting and Its Application to the Three-Dimensional Digital Microfluidics. Appl. Phys. Lett. 2012, $100,081604 / 1-081604 / 3$.

(18) Lee, S.; Lee, S.; Kang, K. Jumping of a Droplet on a Superhydrophobic Surface in AC Electrowetting. J. Vis. 2011, 14 (3), 259-264.

(19) Lapierre, F.; Coffinier, Y.; Boukherroub, R.; Thomy, V. Electro-(de)wetting on Superhydrophobic Surfaces. Langmuir 2013, 29 (44), 13346-13351.

(20) Li, F.; Mugele, F. How to Make Sticky Surfaces Slippery: Contact Angle Hysteresis in Electrowetting with Alternating Voltage. Appl. Phys. Lett. 2008, 92, 244108/1$244108 / 3$.

(21) Lee, S. J.; Hong, J.; Kang, K. H.; Kang, I. S.; Lee, S. J. Electrowetting-Induced Droplet Detachment from Hydrophobic Surfaces. Langmuir 2014, 30 (7), 1805-1811.

(22) Chung, S.; Rhee, K.; Cho, S. Bubble Actuation by Electrowetting-on-Dielectric (EWOD) and Its Applications: A Review. Int.J. Precis. Eng. Manuf. 2010, 11 (6), 991-1006.

(23) Kug Chung, S.; Kwon Cho, S. On-Chip Manipulation of Objects Using Mobile Oscillating Bubbles. J. Micromech. Microeng. 2008, 18 (12), 125024.

(24) Zhao, Y.; Cho, S. K. Micro Air Bubble Manipulation by Electrowetting on Dielectric (EWOD): Transporting, Splitting, Merging and Eliminating of Bubbles. Lab. Chip 2007, 7 (2), 273-280. 
(25) Chung, S. K.; Cho, S. K. Capturing, Carrying, and Releasing of Micro-Objects by ACElectroweting-Actuated Oscillating Bubbles. Solid-State Sens. Actuators Microsyst. IEEE Int. Conf. 2009, 821-824.

(26) Chung, S. K.; Zhao, Y.; Yi, U. C.; Cho, S. K. Micro Bubble Fluidics by EWOD and Ultrasonic Excitation for Micro Bubble Tweezers. Micro Electro Mech. Syst. IEEE $20^{\text {th }}$ Int. Conf. 2007, 31-34.

(27) Chung, S. K.; Kwon, J. O.; Cho, S. K. Manipulation of Micro/Mini-Objects by ACElectrowetting-Actuated Oscillating Bubbles: Capturing, Carrying and Releasing. $J$. Adhes. Sci. Technol. 2012, 26 (12-17), 1965-1983.

(28) Pinchasik, B.-E.; Möhwald, H.; Skirtach, A. G. Mimicking Bubble Use in Nature: Propulsion of Janus Particles due to Hydrophobic-Hydrophilic Interactions. Small 2014, $10(13), 2670-2677$.

(29) Mugele, F. Fundamental Challenges in Electrowetting: From Equilibrium Shapes to Contact Angle Saturation and Drop Dynamics. Soft Matter 2009, 5 (18), 3377-3384.

(30) Quinn, A.; Sedev, R.; Ralston, J. Contact Angle Saturation in Electrowetting. J. Phys. Chem. B 2005, 109, 6268-6275.

(31) Friedman, A. M.; Kennedy, J. W. The Self-Diffusion Coefficients of Potassium, Cesium, Iodide and Chloride Ions in Aqueous Solutions1. J. Am. Chem. Soc. 1955, 77 (17), 4499-4501.

(32) Smidsrod, O.; Storker, T. M. Biopolymer Chemistry; 2008.

(33) Schütz, G. J.; Schindler, H.; Schmidt, T. Single-Molecule Microscopy on Model Membranes Reveals Anomalous Diffusion. Biophys. J. 1997, 73 (2), 1073-1080.

(34) Lee, L.-H. Relationships between Surface Wettability and Glass Temperatures of High Polymers. J. Appl. Polym. Sci. 1968, 12, 719-730.

(35) Snoeijer J H ; Andreotti B Moving Contact Lines: Scales Regimes and Dynamical 
(36) Gao, L.; McCarthy, T. J. Contact Angle Hysteresis Explained. Langmuir 2006, 22 (14), $6234-6237$.

(37) Extrand, C. W. A Thermodynamic Model for Contact Angle Hysteresis. J. Colloid Interface Sci. 1998, 207 (1), 11-19.

(38) Marmur, A. Contact Angle Hysteresis on Heterogeneous Smooth Surfaces. J. Colloid Interface Sci. 1994, 168 (1), 40-46.

(39) Kokhuis, T. J. A.; Naaijkens, B. A.; Juffermans, L. J. M.; Kamp, O.; Versluis, M.; de Jong, N. Secondary Bjerknes Forces Deform Targeted Microbubbles. Ultrason. Symp. IUS IEEE Int. 2012, 1-4.

(40) Marmottant, P.; Hilgenfeldt, S. Controlled Vesicle Deformation and Lysis by Single Oscillating Bubbles. Nature 2003, 423 (6936), 153-156.

(41) Ko, S. H.; Lee, S. J.; Kang, K. H. A Synthetic Jet Produced by Electrowetting-Driven Bubble Oscillations in Aqueous Solution. Appl. Phys. Lett. 2009, 94, 194102/1$194102 / 3$.

(42) Lee, J. H.; Lee, K. H.; Won, J. M.; Rhee, K.; Chung, S. K. Mobile Oscillating Bubble Actuated by AC-Electrowetting-on-Dielectric (EWOD) for Microfluidic Mixing Enhancement. Sens. Actuators Phys. 2012, 182 (0), 153-162.

(43) Pelekasis, N. A.; Gaki, A.; Doinikov, A.; Tsamopoulos, J. A. Secondary Bjerknes Forces between Two Bubbles and the Phenomenon of Acoustic Streamers. J. Fluid Mech. 2004, 500, 313-347.

(44) Verho, T.; Korhonen, J. T.; Sainiemi, L.; Jokinen, V .; Bower, C.; Franze, K.; Franssila, S.; Andrew, P.; Ikkala, O.; Ras, R. H. A. Reversible Switching between Superhydrophobic States on a Hierarchically Structured Surface. Proc. Natl. Acad. Sci. 2012, 109 (26), 10210-10213. 
(45) Papadopoulou, E. L.; Barberoglou, M.; Zorba, V.; Manousaki, A.; Pagkozidis, A.; Stratakis, E.; Fotakis, C. Reversible Photoinduced Wettability Transition of Hierarchical ZnO Structures. J. Phys. Chem. C 2009, 113 (7), 2891-2895.

(46) Barberoglou, M.; Zorba, V.; Pagozidis, A.; Fotakis, C.; Stratakis, E. Electrowetting Properties of Micro/Nanostructured Black Silicon. Langmuir 2010, 26, 13007-13014.

(47) Borkent, B. M.; Schoeherr, H.; Le Caee, G.; Dollet, B.; Lohse, D. Preferred Sizes and Ordering in Surface Nanobubble Populations. Phys. Rev. E Stat. Nonlin. Soft Matter Phys. 2009, 80, 036315/1-036315/4.

(48) Zhang, X. H.; Quinn, A.; Ducker, W. A. Nanobubbles at the Interface between Water and a Hydrophobic Solid. Langmuir 2008, 24 (9), 4756-4764. 